\title{
Exact relations of the quasienergy functional and the exchange-correlation potential from the Floquet formulation of time-dependent density functional theory
}

\author{
Dmitry A. Telnov and Shih-I Chu \\ Department of Chemistry, University of Kansas, and Kansas Center for Advanced Scientific Computing, Lawrence, Kansas 66045
}

(Received 15 July 2000; published 13 December 2000)

\begin{abstract}
In the framework of the Floquet formulation of time-dependent density functional theory we present several exact relations involving different parts of the quasienergy functional. These relations hold when the exact densities and exchange-correlation energy functional are employed. They can be used as useful constraints and tests when searching for the approximate forms of the time-dependent exchange-correlation functionals. The general results are illustrated on an exactly soluble model, Hooke's atom in a linearly polarized monochromatic laser field.
\end{abstract}

DOI: 10.1103/PhysRevA.63.012514

PACS number(s): 71.45.Gm, 71.15.Mb, 31.15.Ew

\section{INTRODUCTION}

Time-independent density functional theory (DFT) of many-electron systems, based on the fundamental works of Hohenberg and Kohn [1] and Kohn and Sham [2], is now a well established and practical tool in various branches of chemistry and physics [3]. Being a formalism of many-body theory in terms of the electron density $\rho(\mathbf{r})$, DFT has proved to be accurate and computationally much less expensive than the $a b$ initio wave functional methods, and this accounts for its great success in time-independent electronic-structure calculations of the ground states of many-electron systems.

To study more interesting dynamical processes, one needs a time-dependent DFT (TDDFT) [4-7]. Runge and Gross [7] have developed a time-dependent Kohn-Sham theory by considering the action to be stationary with respect to the density variations. Several groups have also considered timedependent current density functional theory (TDCDFT) recently $[8-10]$, where the action needs to be stationary with respect to variations in paramagnetic current density as well as the density itself. The central result of the modern TDDFT and TDCDFT is a set of time-dependent Kohn-Sham (TDKS) equations which are structurally similar to the timedependent Hartree-Fock (TDHF) equations but include in principle exactly all many-body effects through a timedependent exchange-correlation potential.

Recently we presented the Floquet formulation of TDDFT [11] and TDCDFT [12] for atoms and molecules in intense periodic and quasiperiodic (multicolor) [13] time-dependent fields, allowing the reduction of TDKS equations to equivalent time-independent Floquet matrix eigenvalue problems. In the Floquet formulation of TDDFT, the main role is played by the quasienergy functional (compare with the action functional in the general time-dependent formulation [7]):

$$
F[\Phi]=\langle\langle\Phi|\hat{\mathcal{H}}| \Phi\rangle\rangle
$$

where $\hat{\mathcal{H}}=H-i \partial / \partial t$ is the Hamiltonian operator in the extended Hilbert space which contains time-periodic wave functions. The inner product in the extended Hilbert space is defined as ( $T$ is the period):

$$
\langle\langle\Phi \mid \Xi\rangle\rangle=\frac{1}{T} \int_{0}^{T} d t\langle\Phi \mid \Xi\rangle .
$$

Variation of the functional (1) under the normalization condition

$$
\langle\langle\Phi \mid \Phi\rangle\rangle=1
$$

leads to the time-dependent Schrödinger equation for the time-periodic multielectron wave function $\Phi$. The solution brings a stationary value (equal to the quasienergy $\varepsilon$ ) to the functional (1). Note that the normalization condition for the function $\Phi$ should be satisfied also in the ordinary Hilbert space at any time moment $t$ :

$$
\langle\Phi(t) \mid \Phi(t)\rangle=1
$$

Equation (4) holds because the Hamiltonian $H$ is a Hermitian operator (in other words, the number of electrons is conserved). The quasienergy functional $F[\Phi]$ can be represented as a sum of contributions from time-dependent quantities such as kinetic energy $T(t)$, single-particle potential energy $U(t)$, electron-electron interaction $V_{e e}(t)$, interaction with the external time-dependent field $V_{\text {ext }}(t)$, and specific term due to the derivative with respect to time $D(t)$ :

$$
F[\Phi]=\frac{1}{T} \int_{0}^{T} d t\left[T(t)+V_{e e}(t)+U(t)+V_{e x t}(t)+D(t)\right],
$$

where

$$
\begin{aligned}
T(t) & =\left\langle\Phi\left|-\frac{1}{2} \sum_{j=1}^{N} \nabla_{j}^{2}\right| \Phi\right\rangle, \\
V_{e e}(t) & =\left\langle\Phi\left|\frac{1}{2} \sum_{i \neq j} \frac{1}{\left|\mathbf{r}_{i}-\mathbf{r}_{j}\right|}\right| \Phi\right\rangle,
\end{aligned}
$$




$$
\begin{gathered}
U(t)=\left\langle\Phi\left|\sum_{j=1}^{N} u\left(\mathbf{r}_{j}\right)\right| \Phi\right\rangle, \\
V_{\text {ext }}(t)=\left\langle\Phi\left|\sum_{j=1}^{N} v_{\text {ext }}\left(\mathbf{r}_{j}, t\right)\right| \Phi\right\rangle, \\
D(t)=\left\langle\Phi\left|-i \frac{\partial}{\partial t}\right| \Phi\right\rangle,
\end{gathered}
$$

$N$ being the number of electrons and $u(\mathbf{r})$ and $v_{\text {ext }}(\mathbf{r}, t)$ the single-particle atomic and external field potentials, respectively. Atomic units will be used throughout this paper.

Since time is treated like any other (coordinate) variable in the extended Hilbert space, one has a steady-state problem for the quasienergy functional (1) and it can be rigorously justified that $\hat{\mathcal{H}}(t), \hat{H}(t), \Phi(t)$, and the quasienergy $\varepsilon$ are all unique functionals of the electron spin densities (see, e.g., [5]). Thus the quasienergy functional Eq. (1) can be expressed as a functional of the spin densities.

Consider the corresponding Kohn-Sham system of noninteracting particles with the same electron spin densities. As the spin densities are periodic in time, the quasienergy solutions of the corresponding time-dependent Schrödinger equations may be sought, and one can introduce the time-periodic Kohn-Sham spin orbitals $\phi_{k}^{\sigma}(\mathbf{r}, t)$ (the superscript $\sigma$ stands for the spin projection, taking values $\alpha$ and $\beta$ for spin up and down, respectively; the subscript $k$ enumerates the orbitals with the same spin). Denoting by $\rho^{\sigma}$ the spin density corresponding to the spin $\sigma$, and by $\rho$ the total density,

$$
\begin{gathered}
\rho^{\sigma}(\mathbf{r}, t)=\sum_{k}\left|\phi_{k}^{\sigma}(\mathbf{r}, t)\right|^{2}, \\
\rho(\mathbf{r}, t)=\sum_{\sigma} \rho^{\sigma}(\mathbf{r}, t),
\end{gathered}
$$

one can rewrite the quasienergy functional (5) in the following form:

$$
\begin{aligned}
F\left[\Phi\left[\rho^{\alpha}, \rho^{\beta}\right]\right] \equiv & F\left[\rho^{\alpha}, \rho^{\beta}\right] \\
= & \frac{1}{T} \int_{0}^{T} d t\left[T_{s}(t)+J(t)+U(t)\right. \\
& \left.+V_{e x t}(t)+D_{s}(t)+E_{x c}(t)\right] .
\end{aligned}
$$

The time-dependent quantities under the integral (13) are defined as follows:

$$
\begin{gathered}
T_{s}(t)=\sum_{k, \sigma}\left\langle\phi_{k}^{\sigma}(\mathbf{r}, t)\left|-\frac{1}{2} \nabla^{2}\right| \phi_{k}^{\sigma}(\mathbf{r}, t)\right\rangle, \\
J(t)=\frac{1}{2} \int d^{3} r \int d^{3} r^{\prime} \frac{\rho(\mathbf{r}, t) \rho\left(\mathbf{r}^{\prime}, t\right)}{\left|\mathbf{r}-\mathbf{r}^{\prime}\right|}, \\
U(t)=\int d^{3} r \rho(\mathbf{r}, t) u(\mathbf{r}),
\end{gathered}
$$

$$
\begin{gathered}
V_{\text {ext }}(t)=\int d^{3} r \rho(\mathbf{r}, t) v_{e x t}(\mathbf{r}, t), \\
D_{s}(t)=\sum_{k, \sigma}\left\langle\phi_{k}^{\sigma}(\mathbf{r}, t)\left|-i \frac{\partial}{\partial t}\right| \phi_{k}^{\sigma}(\mathbf{r}, t)\right\rangle .
\end{gathered}
$$

The single-particle potential expectation value $U(t)$ and external field expectation value $V_{\text {ext }}(t)$ are the same as in the original interacting multi-electron system for the spin densities are the same. The noninteracting kinetic energy $T_{s}(t)$ and classical electron-electron repulsion (Hartree) energy $J(t)$ are different from $T(t)$ and $V_{e e}(t)$, respectively. The difference is taken into account through the exchangecorrelation energy $E_{x c}(t)$ :

$$
E_{x c}(t)=T(t)+V_{e e}(t)+D(t)-T_{s}(t)-J(t)-D_{s}(t) .
$$

The exchange-correlation energy $E_{x c}(t)$ is an unknown functional of the time-dependent spin densities. The simplest approximation for this functional is the adiabatic local-density approximation (ALDA) [4], a straightforward extension of the steady-state LDA to the time-dependent domain, preserving locality in both coordinate space and time. However, it has been inferred that the exact exchange-correlation functional may be nonlocal in both space and time, and several recent attempts have been made to study time nonlocality (memory effects) [14-17].

The purpose of this paper is twofold. First we present several exact relations involving different parts of the quasienergy functional. These relations, which hold in the Floquet formulation of TDDFT when the exact densities and exchange-correlation energy and potential are employed, can be used as constraints and tests when searching for the approximate forms of the time-dependent exchange-correlation functionals. Second, to illustrate the general Floquet TDDFT results, we present an exact solution of the exchangecorrelation energy and potential for a soluble model of twoelectron Hooke's atoms in linearly polarized monochromatic laser fields.

The paper is organized as follows. In Sec. II we establish exact relations regarding the time dependence of various parts of the quasienergy functional (Sec. II A) and based on the virial theorem (Sec. II B). In Sec. III we use the example of an exactly soluble model (Hooke's atom in a linearly polarized monochromatic field) to illustrate the general applicability of the exact relations. The model has some intrinsic limitations since it exhibits the motion prescribed by the harmonic potential theorem, which is only a subset of general time-dependent behavior. However, it is still instructive to test the general formulas in an environment where many analytical results are available.

\section{EXACT RELATIONS IN THE FLOQUET FORMULATION OF TDDFT}

In this section, we study several exact relations regarding the contributions to the quasienergy functional, Eq. (13). The relations involving the exchange-correlation energy and po- 
tential are of primary importance since they can serve as additional constraints in the search for approximate timedependent exchange-correlation functionals. Exact relations or theorems for the time-dependent quantities described above may be established in the framework of the Floquet formulation of TDDFT, just as they hold in the general TDDFT [18]. Since the quasienergy in the Floquet formulation of TDDFT is a time-independent quantity, some additional constraints do exist that are not available in general TDDFT.

\section{A. Time derivatives of kinetic, potential, and exchange- correlation energies}

First, consider a multielectron system and recall that the multielectron time-periodic wave function $\Phi$ satisfies the time-dependent Schrödinger equation

$$
\left[H(t)-i \frac{\partial}{\partial t}\right] \Phi\left(\mathbf{r}_{1}, \ldots, \mathbf{r}_{N}, t\right)=\varepsilon \Phi\left(\mathbf{r}_{1}, \ldots, \mathbf{r}_{N}, t\right) .
$$

Since the normalization condition (4) holds, one can write the following equation for the quasienergy $\varepsilon$ :

$$
\varepsilon=\left\langle\Phi\left|H(t)-i \frac{\partial}{\partial t}\right| \Phi\right\rangle
$$

Note that the inner product on the right-hand side of Eq. (21) is evaluated in the ordinary Hilbert space, so there is no integration with respect to time. That is why the quasienergy $\varepsilon$ can be represented as a sum of time-dependent terms appearing in Eq. (5):

$$
\varepsilon=T(t)+V_{e e}(t)+U(t)+V_{e x t}(t)+D(t) .
$$

The right-hand side of Eq. (22) must be equal to the constant $\varepsilon$ at any time moment. Hence its derivative with respect to time vanishes:

$$
\frac{d}{d t} T(t)+\frac{d}{d t} V_{e e}(t)+\frac{d}{d t} U(t)+\frac{d}{d t} V_{e x t}(t)+\frac{d}{d t} D(t)=0
$$

Equations (22) and (23) express the first exact relations in the Floquet description of a multielectron system subject to a time-periodic external field (no density functional theory so far). The time derivatives of the single-particle potential expectation value $U(t)$ and external field potential $V_{\text {ext }}(t)$ can be expressed as

$$
\begin{gathered}
\frac{d}{d t} U(t)=\int d^{3} r \frac{\partial \rho(\mathbf{r}, t)}{\partial t} u(\mathbf{r}) \\
\frac{d}{d t} V_{e x t}(t)=\int d^{3} r\left[\frac{\partial \rho(\mathbf{r}, t)}{\partial t} v_{e x t}(\mathbf{r}, t)+\rho(\mathbf{r}, t) \frac{\partial v_{e x t}(\mathbf{r}, t)}{\partial t}\right] .
\end{gathered}
$$

On the other hand, differentiating Eq. (21) with respect to time, and taking into account that $\Phi$ is an eigenfunction of the operator $[H(t)-i \partial / \partial t]$ with the eigenvalue $\varepsilon$, one obtains

$$
\begin{array}{r}
\frac{d}{d t} D(t)+\varepsilon\left\langle\frac{\partial \Phi}{\partial t} \mid \Phi\right\rangle+\varepsilon *\left\langle\Phi \mid \frac{\partial \Phi}{\partial t}\right\rangle \\
+\left\langle\Phi\left|\frac{\partial}{\partial t} \sum_{j=1}^{N} v_{e x t}\left(\mathbf{r}_{j}, t\right)\right| \Phi\right\rangle=0 .
\end{array}
$$

Supposing that the quasienergy $\varepsilon$ is real, and taking into account normalization condition (4), one arrives at the relation

$$
\frac{d}{d t} D(t)+\int d^{3} r \rho(\mathbf{r}, t) \frac{\partial v_{e x t}(\mathbf{r}, t)}{\partial t}=0
$$

Making use of this result in Eq. (23) and taking into account Eqs. (24) and (25), the sum of time derivatives of the kinetic energy and electron-electron interaction can be expressed through the integral containing the time derivative of the density and single-particle atomic and external field potentials:

$$
\frac{d}{d t} T(t)+\frac{d}{d t} V_{e e}(t)=-\int d^{3} r \frac{\partial \rho(\mathbf{r}, t)}{\partial t}\left[u(\mathbf{r})+v_{e x t}(\mathbf{r}, t)\right]
$$

Equations (27) and (28) are other important exact relations which hold in the framework of the Floquet formalism.

Now we are going to apply the results to the Floquet formulation of TDDFT. Consider the corresponding KohnSham system of noninteracting particles. The quasienergy Kohn-Sham equation for the time-periodic spin orbital $\phi_{k}^{\sigma}$ reads as

$$
\begin{aligned}
& {\left[-\frac{1}{2} \nabla^{2}+u(\mathbf{r})+v_{H}(\mathbf{r}, t)+v_{x c}(\mathbf{r}, t)+v_{\text {ext }}(\mathbf{r}, t)-i \frac{\partial}{\partial t}\right]} \\
& \quad \times \phi_{k}^{\sigma}=\boldsymbol{\epsilon}_{k}^{\sigma} \phi_{k}^{\sigma} .
\end{aligned}
$$

Here $v_{H}(\mathbf{r}, t)$ is the Hartree potential, $v_{x c}(\mathbf{r}, t)$ is the exchange-correlation potential, and $\epsilon_{k}^{\sigma}$ is the orbital quasienergy. The normalization condition

$$
\left\langle\phi_{k}^{\sigma} \mid \phi_{k}^{\sigma}\right\rangle=1
$$

is assumed. Multiplying Eq. (29) by $\left[\phi_{k}^{\sigma}\right]^{*}$ and taking the integral with respect to the coordinate $\mathbf{r}$, one obtains

$$
\begin{aligned}
\left\langle\phi_{k}^{\sigma}\right| & -\frac{1}{2} \nabla^{2}+u(\mathbf{r})+v_{H}(\mathbf{r}, t)+v_{x c}(\mathbf{r}, t)+v_{\text {ext }}(\mathbf{r}, t)\left|\phi_{k}^{\sigma}\right\rangle \\
& +\left\langle\phi_{k}^{\sigma}\left|-i \frac{\partial}{\partial t}\right| \phi_{k}^{\sigma}\right\rangle=\epsilon_{k}^{\sigma} .
\end{aligned}
$$

Performing a summation of Eq. (31) over all spin orbitals results in the following relation that must be satisfied at arbitrary time moment $t$ : 


$$
\begin{aligned}
T_{s}(t)+D_{s}(t)+\int & d^{3} r \rho(\mathbf{r}, t)\left[u(\mathbf{r})+v_{H}(\mathbf{r}, t)+v_{x c}(\mathbf{r}, t)\right. \\
\left.+v_{e x t}(\mathbf{r}, t)\right]= & T_{s}(t)+2 J(t)+U(t)+V_{e x t}(t)+D_{s}(t) \\
& +\int d^{3} r \rho(\mathbf{r}, t) v_{x c}(\mathbf{r}, t)=\sum_{k, \sigma} \epsilon_{k}^{\sigma} .
\end{aligned}
$$

Here $T_{s}(t)$ and $D_{s}(t)$ are the kinetic energy and timederivative expectation values for the noninteracting KohnSham system, respectively:

$$
\begin{aligned}
& T_{s}(t)=\sum_{k, \sigma}\left\langle\phi_{k}^{\sigma}\left|-\frac{1}{2} \nabla^{2}\right| \phi_{k}^{\sigma}\right\rangle, \\
& D_{s}(t)=\sum_{k, \sigma}\left\langle\phi_{k}^{\sigma}\left|-i \frac{\partial}{\partial t}\right| \phi_{k}^{\sigma}\right\rangle .
\end{aligned}
$$

Differentiating Eq. (32) with respect to time, one obtains

$$
\begin{aligned}
& \frac{d}{d t} T_{s}(t)+\frac{d}{d t} D_{s}(t)+\int d^{3} r \frac{\partial \rho(\mathbf{r}, t)}{\partial t}\left[v_{H}(\mathbf{r}, t)+v_{x c}(\mathbf{r}, t)\right. \\
& \left.+v_{e x t}(\mathbf{r}, t)+u(\mathbf{r})\right]+\int d^{3} r \rho(\mathbf{r}, t) \\
& \quad \times\left[\frac{\partial v_{H}(\mathbf{r}, t)}{\partial t}+\frac{\partial v_{x c}(\mathbf{r}, t)}{\partial t}+\frac{\partial v_{e x t}(\mathbf{r}, t)}{\partial t}\right]=0
\end{aligned}
$$

On the other hand, differentiating Eq. (31) with respect to time and following the steps described above that led to Eq. (27) for the multielectron system, one arrives at the following relation:

$$
\begin{gathered}
\frac{d}{d t}\left\langle\boldsymbol{\phi}_{k}^{\sigma}\left|-i \frac{\partial}{\partial t}\right| \boldsymbol{\phi}_{k}^{\sigma}\right\rangle+\left\langle\boldsymbol{\phi}_{k}^{\sigma}\right| \frac{\partial v_{H}(\mathbf{r}, t)}{\partial t}+\frac{\partial v_{x c}(\mathbf{r}, t)}{\partial t} \\
+\frac{\partial v_{\text {ext }}(\mathbf{r}, t)}{\partial t}\left|\phi_{k}^{\sigma}\right\rangle=0 .
\end{gathered}
$$

After summation over all spin orbitals, Eq. (36) takes the form

$$
\begin{aligned}
& \frac{d}{d t} D_{s}(t)+\int d^{3} r \rho(\mathbf{r}, t) \\
& \quad \times\left[\frac{\partial v_{H}(\mathbf{r}, t)}{\partial t}+\frac{\partial v_{x c}(\mathbf{r}, t)}{\partial t}+\frac{\partial v_{e x t}(\mathbf{r}, t)}{\partial t}\right]=0 .
\end{aligned}
$$

Substituting Eq. (37) into Eq. (35), the following expression is obtained:

$$
\begin{aligned}
\frac{d}{d t} T_{s}(t)= & -\int d^{3} r \frac{\partial \rho(\mathbf{r}, t)}{\partial t}\left[u(\mathbf{r})+v_{H}(\mathbf{r}, t)+v_{x c}(\mathbf{r}, t)\right. \\
& \left.+v_{\text {ext }}(\mathbf{r}, t)\right] .
\end{aligned}
$$

Equations (37) and (38) are exact relations that express the dependence of the single-particle kinetic energy and time- derivative operator expectation values on time through the density and potentials, including the exchange-correlation potential.

Now we consider the most important relations involving the time-dependent exchange-correlation energy and potential. Taking into account the definition of the exchangecorrelation energy (19) and Eq. (22), the relation (32) can be rewritten in the following form:

$$
E_{x c}(t)=\varepsilon-\sum_{k, \sigma} \epsilon_{k}^{\sigma}+J(t)+\int d^{3} r \rho(\mathbf{r}, t) v_{x c}(\mathbf{r}, t) .
$$

The exchange-correlation energy $E_{x c}(t)$ itself can be expressed through the expectation value of the exchangecorrelation potential, Hartree energy $J(t)$, total quasienergy $\varepsilon$, and Kohn-Sham spin-orbital quasienergies $\epsilon_{k}^{\sigma}$. Differentiating Eq. (39) with respect to time, one obtains an equation that does not contain quasienergies and relates the time dependence of the exchange-correlation energy directly to that of the exchange-correlation potential expectation value and Hartree energy:

$$
\frac{d}{d t} E_{x c}(t)=\frac{d}{d t} \int d^{3} r \rho(\mathbf{r}, t) v_{x c}(\mathbf{r}, t)+\frac{d}{d t} J(t) .
$$

Equations (39) and (40) are the main results of this section. Equation (40) can serve as a constraint in the search for approximate time-dependent exchange-correlation functionals since it establishes a relation between the time derivatives of the exchange-correlation energy $E_{x c}(t)$ and the exchangecorrelation potential $v_{x c}(\mathbf{r}, t)$, which is supposed to be a functional derivative of $(1 / T) \int_{0}^{T} d t E_{x c}(t)$ with respect to the density.

Note that our result (40) for the time derivative of the exchange-correlation energy differs from that obtained in Ref. [18]. This is because of the different definition of the exchange-correlation energy. In Ref. [18] the exchangecorrelation energy (let us denote it $\widetilde{E}_{x c}$ ) is defined as in timeindependent DFT; the time-derivative contributions are not taken into account:

$$
\widetilde{E}_{x c}(t)=T(t)+V_{e e}(t)-T_{s}(t)-J(t) .
$$

For the time derivative of this quantity the same result as in Ref. [18] is obtained:

$$
\frac{d}{d t} \widetilde{E}_{x c}(t)=\int d^{3} r \frac{\partial \rho(\mathbf{r}, t)}{\partial t} v_{x c}(\mathbf{r}, t)
$$

However, it is $E_{x c}(t)$ and not $\widetilde{E}_{x c}(t)$ that is present in the quasienergy functional (13) of the Kohn-Sham system and makes it equal to the quasienergy of the original multielectron system. The exchange-correlation potential $v_{x c}(\mathbf{r}, t)$ is a functional derivative with respect to the density of that part of the functional (13) which contains $E_{x c}(t)$. 


\section{B. Virial theorem}

The virial theorem in the Floquet formulation of timedependent density functional theory can be obtained by straightforward generalization of that in traditional quantum mechanics. Consider first a multielectron system. It follows from Eq. (20) that the expectation value of the operator

$$
A=-\frac{i}{2} \sum_{j=1}^{N}\left[\left(\mathbf{r}_{j} \cdot \nabla_{j}\right)+\left(\nabla_{j} \cdot \mathbf{r}_{j}\right)\right]
$$

satisfies the following equation [Eqs. (6), (7), and (8) are also taken into account]:

$$
\begin{aligned}
\frac{d}{d t}\langle\Phi|A| \Phi\rangle= & 2\left\langle\Phi\left|-\frac{1}{2} \sum_{j=1}^{N} \nabla_{j}^{2}\right| \Phi\right\rangle \\
& -\left\langle\Phi\left|\sum_{j=1}^{N}\left[\mathbf{r}_{j} \cdot \nabla_{j} u\left(\mathbf{r}_{j}\right)\right]\right| \Phi\right\rangle \\
& -\left\langle\Phi\left|\sum_{j=1}^{N}\left[\mathbf{r}_{j} \cdot \nabla_{j} v_{e x t}\left(\mathbf{r}_{j}, t\right)\right]\right| \Phi\right\rangle \\
& -\left\langle\Phi\left|\sum_{j=1}^{N}\left(\mathbf{r}_{j} \cdot \nabla_{j} \frac{1}{2} \sum_{\substack{i=1 \\
i \neq j}}^{N} \frac{1}{\left|\mathbf{r}_{i}-\mathbf{r}_{j}\right|}\right)\right| \Phi\right\rangle
\end{aligned}
$$

where $\Phi$ is the time-periodic multielectron wave function Eq. (20). Making use of Eq. (6) and the homogeneity properties of the Coulomb potential, Eq. (44) can be rewritten in terms of the quantities $T(t)$ and $V_{e e}(t)$ :

$$
\begin{aligned}
\frac{d}{d t}\langle\Phi|A| \Phi\rangle= & 2 T(t)+V_{e e}(t)-\left\langle\Phi\left|\sum_{j=1}^{N}\left[\mathbf{r}_{j} \cdot \nabla_{j} u\left(\mathbf{r}_{j}\right)\right]\right| \Phi\right\rangle \\
& -\left\langle\Phi\left|\sum_{j=1}^{N}\left[\mathbf{r}_{j} \cdot \nabla_{j} v_{e x t}\left(\mathbf{r}_{j}, t\right)\right]\right| \Phi\right\rangle
\end{aligned}
$$

On the other hand, the expectation value of $A$ is the time derivative of the squared distance expectation value:

$$
\langle\Phi|A| \Phi\rangle=\frac{1}{2} \frac{d}{d t}\left\langle\Phi\left|\sum_{j=1}^{N} r_{j}^{2}\right| \Phi\right\rangle
$$

Inserting Eq. (46) in the left-hand side of Eq. (45), one obtains

$$
\begin{aligned}
\frac{1}{2} \frac{d^{2}}{d t^{2}}\left\langle\Phi\left|\sum_{j=1}^{N} r_{j}^{2}\right| \Phi\right\rangle= & 2 T(t)+V_{e e}(t) \\
& -\left\langle\Phi\left|\sum_{j=1}^{N}\left[\mathbf{r}_{j} \cdot \nabla_{j} u\left(\mathbf{r}_{j}\right)\right]\right| \Phi\right\rangle \\
& -\left\langle\Phi\left|\sum_{j=1}^{N}\left[\mathbf{r}_{j} \cdot \nabla_{j} v_{\text {ext }}\left(\mathbf{r}_{j}, t\right)\right]\right| \Phi\right\rangle
\end{aligned}
$$

Performing the same analysis for the noninteracting Kohn-Sham system, one arrives at the following relation:

$$
\begin{aligned}
\frac{1}{2} \frac{d^{2}}{d t^{2}} \sum_{k, \sigma}\left\langle\phi_{k}^{\sigma}\left|r^{2}\right| \phi_{k}^{\sigma}\right\rangle \\
=2 T_{s}(t)-\sum_{k, \sigma}\left\langle\phi_{k}^{\sigma}|[\mathbf{r} \cdot \nabla u(\mathbf{r})]| \phi_{k}^{\sigma}\right\rangle+J(t) \\
\quad-\sum_{k, \sigma}\left\langle\phi_{k}^{\sigma}\left|\left[\mathbf{r} \cdot \nabla v_{x c}(\mathbf{r}, t)\right]\right| \phi_{k}^{\sigma}\right\rangle \\
-\sum_{k, \sigma}\left\langle\phi_{k}^{\sigma}\left|\left[\mathbf{r} \cdot \nabla v_{\text {ext }}(\mathbf{r}, t)\right]\right| \phi_{k}^{\sigma}\right\rangle .
\end{aligned}
$$

The left-hand sides of Eqs. (47) and (48) as well as the contributions due to the single-particle potential $u(\mathbf{r})$ and external field $v_{\text {ext }}(\mathbf{r}, t)$ coincide, since the multielectron and Kohn-Sham systems possess the same density. Consequently one obtains from combining Eqs. (47) and (48)

$$
\begin{aligned}
2 T(t)+V_{e e}(t)= & 2 T_{s}(t)+J(t) \\
& -\sum_{k, \sigma}\left\langle\phi_{k}^{\sigma}\left|\left[\mathbf{r} \cdot \nabla v_{x c}(\mathbf{r}, t)\right]\right| \phi_{k}^{\sigma}\right\rangle .
\end{aligned}
$$

With the help of the expression for the exchange-correlation energy (19), Eq. (49) can be recast in the final form

$$
E_{x c}(t)+T_{c}(t)-D_{c}(t)=-\int d^{3} r \rho(\mathbf{r}, t)\left[\mathbf{r} \cdot \nabla v_{x c}(\mathbf{r}, t)\right],
$$

where the correlation kinetic energy $T_{c}(t)$ and correlation time derivative $D_{c}(t)$ are defined as

$$
\begin{gathered}
T_{c}(t)=T(t)-T_{s}(t), \\
D_{c}(t)=D(t)-D_{s}(t) .
\end{gathered}
$$

Equation (50) is the main result of the current subsection. It may serve as an additional constraint when searching for approximate forms of the time-dependent exchangecorrelation energy functional and potential. Again, our result (50) differs from the analogous expression in Ref. [18] due to different definition of the exchange-correlation energy. There is no term $D_{c}(t)$ in the theorem of Ref. [18].

The general results of this section will be illustrated in the next section on the field-driven Hooke's atom model (two electrons interacting with the Coulomb force and bound in a one-particle harmonic oscillator potential).

\section{A MODEL STUDY: HOOKE'S ATOM IN A LINEARLY POLARIZED LASER FIELD}

The Hooke's atom model has often been used in timeindependent DFT studies. Since the exact (in some cases analytic) solution of the two-electron problem is available in this model, it allows for the construction of an exact exchange-correlation potential. That is why this model ap- 
peared so attractive in testing various approximate forms of exchange-correlation potential. Extensive tests of approximate functionals in the time-independent DFT were performed in Refs. [19] and [20], and analytic solutions were investigated in Refs. [21-23]. We consider here the extension of the Hooke's atom model to the time domain.

The unperturbed Hamiltonian $H_{0}$ of the two-electron Hooke's atom is as follows (in atomic units):

$$
H_{0}=-\frac{1}{2} \nabla_{1}^{2}-\frac{1}{2} \nabla_{2}^{2}+\frac{1}{\left|\mathbf{r}_{2}-\mathbf{r}_{1}\right|}+\frac{1}{2} \omega_{0}^{2}\left(r_{1}^{2}+r_{2}^{2}\right) .
$$

In the presence of a time-dependent external field with electric field amplitude $\mathcal{E}(t)$, the interaction of the electrons with the external field in the dipole approximation reads as

$$
H_{1}=-\left[\mathcal{E}(t) \cdot \mathbf{r}_{1}\right]-\left[\mathcal{E}(t) \cdot \mathbf{r}_{2}\right] .
$$

The total Hamiltonian $H$ is defined as the sum of $H_{0}$ and $H_{1}$ :

$$
H=H_{0}+H_{1} .
$$

The time-dependent Schrödinger equation for the twoelectron wave function $\Psi\left(\mathbf{r}_{1}, \mathbf{r}_{2}, t\right)$ can be written as

$$
i \frac{\partial}{\partial t} \Psi\left(\mathbf{r}_{1}, \mathbf{r}_{2}, t\right)=H \Psi\left(\mathbf{r}_{1}, \mathbf{r}_{2}, t\right) .
$$

Introducing the center-of-mass and relative coordinates

$$
\begin{gathered}
\mathbf{R}_{C M}=\frac{1}{2}\left(\mathbf{r}_{1}+\mathbf{r}_{2}\right), \\
\mathbf{R}_{R M}=\mathbf{r}_{2}-\mathbf{r}_{1},
\end{gathered}
$$

one can represent the Hamiltonian $H$ in the following separable form:

$$
\begin{aligned}
H= & {\left[-\frac{1}{4} \nabla_{R_{C M}}^{2}+\omega_{0}^{2} R_{C M}^{2}-2\left[\mathcal{E}(t) \cdot \mathbf{R}_{C M}\right]\right] } \\
& +\left[-\nabla_{R_{R M}}^{2}+\frac{1}{R_{R M}}+\frac{1}{4} \omega_{0}^{2} R_{R M}^{2}\right] .
\end{aligned}
$$

If the external field $\mathcal{E}(t)$ is periodic in time, the quasienergy solution of Eq. (56) can be sought. Taking into account the separability of the Hamiltonian (58), the wave function $\Psi\left(\mathbf{r}_{1}, \mathbf{r}_{2}, t\right)$ can be represented as

$$
\Psi\left(\mathbf{r}_{1}, \mathbf{r}_{2}, t\right)=\exp (-i \varepsilon t) \Phi\left(\mathbf{R}_{C M}, t\right) \varphi\left(\mathbf{R}_{R M}, t\right),
$$

where $\varepsilon$ is the quasienergy, and $\Phi\left(\mathbf{R}_{C M}, t\right)$ and $\varphi\left(\mathbf{R}_{R M}, t\right)$ are periodic functions of time. They satisfy the following center-of-mass motion (CM) and relative motion (RM) equations, respectively:

$$
\begin{aligned}
& {\left[-\frac{1}{4} \nabla_{R_{C M}}^{2}-i \frac{\partial}{\partial t}+\omega_{0}^{2} R_{C M}^{2}-2\left[\mathcal{E}(t) \cdot \mathbf{R}_{C M}\right]\right] \Phi\left(\mathbf{R}_{C M}, t\right)} \\
& \quad=\varepsilon_{C M} \Phi\left(\mathbf{R}_{C M}, t\right)
\end{aligned}
$$

$$
\begin{aligned}
& {\left[-\nabla_{R_{R M}}^{2}-i \frac{\partial}{\partial t}+\frac{1}{R_{R M}}+\frac{1}{4} \omega_{0}^{2} R_{R M}^{2}\right] \varphi\left(\mathbf{R}_{R M}, t\right)} \\
& \quad=\varepsilon_{R M} \varphi\left(\mathbf{R}_{R M}, t\right) .
\end{aligned}
$$

Here $\varepsilon_{C M}$ and $\varepsilon_{R M}$ are the CM and RM quasienergies, respectively. The total quasienergy $\varepsilon$ is the sum of the $\mathrm{CM}$ and RM quasienergies:

$$
\varepsilon=\varepsilon_{C M}+\varepsilon_{R M} .
$$

Since the Hamiltonian in Eq. (61) is time independent, a time-independent solution $\varphi\left(\mathbf{R}_{R M}\right)$ exists that satisfies the time-independent Schrödinger equation:

$$
\left[-\nabla_{R_{R M}}^{2}+\frac{1}{R_{R M}}+\frac{1}{4} \omega_{0}^{2} R_{R M}^{2}\right] \varphi\left(\mathbf{R}_{R M}\right)=\varepsilon_{R M} \varphi\left(\mathbf{R}_{R M}\right) .
$$

For the ground state, the eigenfunction $\varphi\left(\mathbf{R}_{R M}\right)$ is spherically symmetric, $\varphi\left(\mathbf{R}_{R M}\right)=\varphi\left(R_{R M}\right)$.

Let the field $\mathcal{E}(t)$ be linearly polarized and monochromatic:

$$
\mathcal{E}(t)=\mathbf{F} \cos \omega t
$$

Then Eq. (60) can be recast in the infinite set of timeindependent equations for the Fourier components $\Phi_{m}\left(\mathbf{R}_{C M}\right)$ of the periodic wave function $\Phi\left(\mathbf{R}_{C M}, t\right)$ :

$$
\begin{gathered}
\Phi\left(\mathbf{R}_{C M}, t\right)=\sum_{m=-\infty}^{\infty} \exp (-i m \omega t) \Phi_{m}\left(\mathbf{R}_{C M}\right), \\
{\left[-\frac{1}{4} \nabla_{R_{C M}}^{2}+\omega_{0}^{2} R_{C M}^{2}\right] \Phi_{m}\left(\mathbf{R}_{C M}\right)-2\left(\mathbf{F} \cdot \mathbf{R}_{C M}\right)\left[\Phi_{m-1}\left(\mathbf{R}_{C M}\right)\right.} \\
\left.+\Phi_{m+1}\left(\mathbf{R}_{C M}\right)\right]=\left(\varepsilon_{C M}+m \omega\right) \Phi_{m}\left(\mathbf{R}_{C M}\right) .
\end{gathered}
$$

An analytic solution of Eq. (60) or Eq. (66) exists; see Sec. III B for details.

The one-electron time-dependent density $\rho(\mathbf{r}, t)$ of the field-driven ground state of the Hooke's atom is calculated according to the following definition:

$$
\rho(\mathbf{r}, t)=2 \int d^{3} r^{\prime}\left|\Phi\left(\frac{1}{2}\left(\mathbf{r}+\mathbf{r}^{\prime}\right), t\right)\right|^{2} \varphi^{2}\left(\left|\mathbf{r}-\mathbf{r}^{\prime}\right|\right) .
$$

Here $\Phi\left(\mathbf{R}_{C M}, t\right)$ and $\varphi\left(R_{R M}\right)$ are the normalized solutions of Eqs. (60) and (63), respectively. The function $\varphi\left(R_{R M}\right)$ is the RM ground state eigenfunction, whereas $\Phi\left(\mathbf{R}_{C M}, t\right)$ is the quasienergy wave function, which reduces to the $\mathrm{CM}$ ground state as the external field is switched off.

\section{A. Unperturbed Hooke's atom}

The unperturbed two-electron Hooke's atom has been investigated several times in the literature [19-23]. Besides presenting some additional results (to our knowledge, the analytic expressions for the Hartree and single-particle potentials for $\omega_{0}=1 / 2$ have not been published yet), we include 
this subsection because it is relevant to the development of the time-dependent theory in the following subsections III B and III C.

Without the external field $(F=0)$, Eq. (60) reduces to the three-dimensional (3D) harmonic oscillator eigenvalue problem. The normalized ground state wave function $\Phi^{(0)}\left(R_{C M}\right)$ is time independent and spherically symmetric and has the well-known expression

$$
\Phi^{(0)}\left(R_{C M}\right)=\left(\frac{2 \omega_{0}}{\pi}\right)^{3 / 4} \exp \left(-\omega_{0} R_{C M}^{2}\right)
$$

and the unperturbed eigenvalue $\varepsilon_{C M}^{(0)}$ is given by

$$
\varepsilon_{C M}^{(0)}=\frac{3}{2} \omega_{0} .
$$

Making use of the CM oscillator eigenfunction (68), one can perform analytically some integrations in the definition of the one-electron density (67) and express the ground state one-electron density of the unperturbed Hooke's atom $\rho^{(0)}(r)$, which also appears spherically symmetric, through a one-dimensional integral containing the normalized RM ground state eigenfunction $\varphi[19]$ :

$$
\begin{aligned}
\rho^{(0)}(r)= & \frac{8}{r}\left(\frac{2 \omega_{0}}{\pi}\right)^{1 / 2} \exp \left(-2 \omega_{0} r^{2}\right) \int_{0}^{\infty} d r^{\prime} r^{\prime} \varphi^{2}\left(r^{\prime}\right) \\
& \times \exp \left(-\frac{1}{2} \omega_{0} r^{\prime 2}\right) \sinh \left(2 \omega_{0} r r^{\prime}\right) .
\end{aligned}
$$

The time-independent Kohn-Sham orbital $\phi_{K S}^{(0)}(r)$ for this two-electron system is obtained immediately from the known density (70),

$$
\phi_{K S}^{(0)}(r)=\left[\frac{1}{2} \rho^{(0)}(r)\right]^{1 / 2},
$$

and the noninteracting kinetic energy $T_{s}^{(0)}$ and the Hartree energy $J^{(0)}$ are calculated as follows:

$$
\begin{gathered}
T_{S}^{(0)}=2\left\langle\phi_{K S}^{(0)}\left|-\frac{1}{2} \nabla^{2}\right| \phi_{K S}^{(0)}\right\rangle, \\
J^{(0)}=\frac{1}{2} \int d^{3} r \rho^{(0)}(r) v_{H}^{(0)}(r) .
\end{gathered}
$$

Here the unperturbed Hartree potential $v_{H}^{(0)}(r)$ has its usual definition:

$$
v_{H}^{(0)}(r)=\int d^{3} r^{\prime} \frac{\rho^{(0)}\left(r^{\prime}\right)}{\left|\mathbf{r}-\mathbf{r}^{\prime}\right|} .
$$

The unperturbed exchange-correlation energy $E_{x c}^{(0)}$ and correlation kinetic energy $T_{c}^{(0)}$ are also calculated according to their conventional definitions:

$$
\begin{gathered}
E_{x c}^{(0)}=T^{(0)}+V_{e e}^{(0)}-T_{s}^{(0)}-J^{(0)}, \\
T_{c}^{(0)}=T^{(0)}-T_{s}^{(0)} .
\end{gathered}
$$

Here $T^{(0)}$ and $V_{e e}^{(0)}$ are the unperturbed kinetic energy and electron-electron repulsion of the two-electron system calculated according to Eqs. (6) and (7), respectively, without external field.

Since for the two-electron system the exact exchange (Hartree-Fock) functional is expressed through the Hartree energy $J^{(0)}$, one can separate the exchange and correlation contributions as follows:

$$
E_{x}^{(0)}=-\frac{1}{2} J^{(0)},
$$

$$
E_{c}^{(0)}=E_{x c}^{(0)}-E_{x}^{(0)}
$$

The Kohn-Sham orbital $\phi_{K S}^{(0)}(r)$ defined by Eq. (71) must satisfy the time-independent Kohn-Sham equation:

$$
\left[-\frac{1}{2} \nabla^{2}+\frac{1}{2} \omega_{0}^{2} r^{2}+v_{s}^{(0)}(r)\right] \phi_{K S}^{(0)}(r)=\epsilon_{K S}^{(0)} \phi_{K S}^{(0)}(r) .
$$

Here $\epsilon_{K S}^{(0)}$ is the orbital energy, and $v_{s}^{(0)}(r)$ is a single-particle potential that includes the Hartree potential $v_{H}^{(0)}(r)$ and exchange-correlation potential $v_{x c}^{(0)}(r)$ :

$$
v_{s}^{(0)}(r)=v_{H}^{(0)}(r)+v_{x c}^{(0)}(r)
$$

With the help of Eqs. (71) and (79), one can obtain the expression for the potential $v_{s}^{(0)}(r)$ through the density $\rho^{(0)}(r)$ :

$$
\begin{aligned}
v_{s}^{(0)}(r)= & \frac{1}{2}\left[\frac{1}{r \rho^{(0)}} \frac{d \rho^{(0)}}{d r}+\frac{1}{2 \rho^{(0)}} \frac{d^{2} \rho^{(0)}}{d r^{2}}\right. \\
& \left.+\left(\frac{1}{2 \rho^{(0)}} \frac{d \rho^{(0)}}{d r}\right)^{2}\right]-\frac{1}{2} \omega_{0}^{2} r^{2}+\epsilon_{K S}^{(0)} .
\end{aligned}
$$

The exchange-correlation potential $v_{x c}^{(0)}(r)$ satisfies the following relation, which can be obtained from the Kohn-Sham equation (79) and the definition of the exchange-correlation energy [a particular case of the general time-dependent relation (39)]:

$$
\int d^{3} r \rho(r) v_{x c}(r)=2 \epsilon_{K S}^{(0)}-\varepsilon^{(0)}+E_{x c}^{(0)}-J^{(0)},
$$

where $\varepsilon^{(0)}$ is the unperturbed total energy of the Hooke's atom.

For some particular values of $\omega_{0}$, the RM equation (63) has analytic solutions [21-23]. Then the unperturbed Hooke's atom problem has a completely analytic solution. For example, this is the case for the ground state eigenfunction and $\omega_{0}=1 / 2$. Below we consider this special case in more detail and we present an analytic expression for the exchange-correlation potential. The expressions for the RM eigenfunction $\varphi\left(R_{R M}\right)$ and eigenvalue $\varepsilon_{R M}$ appear as follows [22]: 


$$
\begin{gathered}
\varphi\left(R_{R M}\right)=[4 \pi(8+5 \sqrt{\pi})]^{-1 / 2}\left(1+\frac{1}{2} R_{R M}\right) \\
\times \exp \left(-\frac{1}{8} R_{R M}^{2}\right), \\
\varepsilon_{R M}=\frac{5}{2} \omega_{0}=\frac{5}{4} .
\end{gathered}
$$

The total wave function $\Psi^{(0)}\left(\mathbf{r}_{1}, \mathbf{r}_{2}, t\right)$ in the original coordinates and the total energy $\varepsilon^{(0)}$ read as

$$
\begin{gathered}
\Psi^{(0)}\left(\mathbf{r}_{1}, \mathbf{r}_{2}, t\right)=\pi^{-3 / 4}[4 \pi(8+5 \sqrt{\pi})]^{-1 / 2}\left[1+\frac{1}{2}\left|\mathbf{r}_{1}-\mathbf{r}_{2}\right|\right] \\
\times \exp \left(-i \varepsilon^{(0)} t-\frac{1}{4}\left(r_{1}^{2}+r_{2}^{2}\right)\right) \\
\varepsilon^{(0)}=2
\end{gathered}
$$

The unperturbed two-electron kinetic energy $T^{(0)}$, oneparticle potential energy $U^{(0)}$, and electron-electron interaction $V_{e e}^{(0)}$ contributions to the total energy $\varepsilon^{(0)}$ are as follows:

$$
T^{(0)}=\frac{10+7 \sqrt{\pi}}{16+10 \sqrt{\pi}}=0.6644176
$$

$$
\begin{aligned}
& U^{(0)}=\frac{14+9 \sqrt{\pi}}{16+10 \sqrt{\pi}}=0.8881392, \\
& V_{e e}^{(0)}=\frac{8+4 \sqrt{\pi}}{16+10 \sqrt{\pi}}=0.4474432 .
\end{aligned}
$$

The one-electron density calculated from the wave function (85) can be written in the following form [22]:

$$
\begin{aligned}
\rho^{(0)}(r)= & \frac{2 \pi^{-3 / 2}}{8+5 \sqrt{\pi}} \exp \left(-\frac{1}{2} r^{2}\right)\left[\frac{1}{4} \sqrt{\frac{\pi}{2}}\left(7+r^{2}\right)\right. \\
& \left.+\exp \left(-\frac{1}{2} r^{2}\right)+\left(r+\frac{1}{r}\right) \sqrt{\frac{\pi}{2}} \operatorname{erf}\left(\frac{r}{\sqrt{2}}\right)\right]
\end{aligned}
$$

As an additional contribution, we present here the analytic expressions for the Hartree potential $v_{H}^{(0)}(r)$ and the singleparticle potential $v_{s}^{(0)}(r)$ produced by the density $(90)$ :

$$
\begin{aligned}
& v_{H}^{(0)}(r)= \frac{8 \pi^{-1 / 2}}{8+5 \sqrt{\pi}}\left(\frac{1}{r}\left[\frac{5 \pi}{4} \operatorname{erf}\left(\frac{r}{\sqrt{2}}\right)+2 \sqrt{\pi} \operatorname{erf}(r)\right]-\frac{1}{4} \sqrt{\frac{\pi}{2}} \exp \left(-\frac{1}{2} r^{2}\right)\right. \\
&\left.+\frac{\pi}{2}\left\{1-\left[\operatorname{erf}\left(\frac{r}{\sqrt{2}}\right)\right]^{2}\right\}-\frac{3}{r} \exp \left(-\frac{1}{2} r^{2}\right) \sqrt{\frac{\pi}{2}} \operatorname{erf}\left(\frac{r}{\sqrt{2}}\right)\right), \\
& v_{s}^{(0)}(r)=\frac{1}{4} \frac{5 \sqrt{\pi / 2}+\exp \left(-\frac{1}{2} r^{2}\right)+(r+5 / r) \sqrt{\pi / 2} \operatorname{erf}(r / \sqrt{2})}{(1 / 4) \sqrt{\pi / 2}\left(7+r^{2}\right)+\exp \left(-\frac{1}{2} r^{2}\right)+(r+1 / r) \sqrt{\pi / 2} \operatorname{erf}(r / \sqrt{2})} \\
&-\frac{1}{8}\left[\frac{(1 / 2) \sqrt{\pi / 2} r+(1 / r) \exp \left(-\frac{1}{2} r^{2}\right)+\left(1-1 / r^{2}\right) \sqrt{\pi / 2} \operatorname{erf}(r / \sqrt{2})}{(1 / 4) \sqrt{\pi / 2}\left(7+r^{2}\right)+\exp \left(-\frac{1}{2} r^{2}\right)+(r+1 / r) \sqrt{\pi / 2} \operatorname{erf}(r / \sqrt{2})}\right]^{2} .
\end{aligned}
$$

The potential $v_{s}^{(0)}(r)$ has correct asymptotic behavior as $r$ $\rightarrow \infty$ (it vanishes as $1 / r$ ). The orbital energy value $\epsilon_{K S}^{(0)}$ obtained from Eq. (81) under the condition that the potential $v_{s}^{(0)}(r)$ vanishes at infinity is equal to 5/4. Generally, it can be proved that $\epsilon_{K S}^{(0)}$ is equal to $\varepsilon_{R M}$ [23]. The analytic expression for the exchange-correlation potential $v_{x c}^{(0)}(r)$ follows immediately from Eqs. (91) and (92):

$$
v_{x c}^{(0)}(r)=v_{s}^{(0)}(r)-v_{H}^{(0)}(r)
$$

The numerical values of various quantities defined in Eqs. (72), (73), (75), (76), (77), and (78) for the case of $\omega_{0}$ $=1 / 2$ are

$$
\begin{aligned}
& T_{s}^{(0)}=0.6352457, \\
& T_{c}^{(0)}=0.02917195, \\
& J^{(0)}=1.0302504,
\end{aligned}
$$




$$
\begin{aligned}
& E_{x c}^{(0)}=-0.5536352, \\
& E_{x}^{(0)}=-0.5151252, \\
& E_{c}^{(0)}=-0.03851005 .
\end{aligned}
$$

As one can see, the correlation energy is quite small compared with the exchange energy. From Eqs. (95) and (99) one can conclude that the correlation contributions to the kinetic and potential energies have opposite signs, and the potential correlation energy is more than two times larger in absolute value.

The expectation value of the exchange-correlation potential (82) can be evaluated without actual computation since all the quantities on the right-hand side of Eq. (82) are known (see discussion above):

$$
\int d^{3} r \rho(r) v_{x c}^{(0)}(r)=-1.0838856 .
$$

Numerical computation of the integral using the analytic expressions (90) and (93), (91), and (92) confirms this result. Using the decomposition of the exchange-correlation potential $v_{x c}^{(0)}(r)$ into the Hartree-Fock exchange potential [which is equal to $\left.-(1 / 2) v_{H}^{(0)}(r)\right]$ and the correlation potential $v_{c}^{(0)}(r)$, one obtains the following relation for the correlation potential $v_{c}^{(0)}(r)$ :

$$
\int d^{3} r \rho(r) v_{c}^{(0)}(r)=-0.05363524
$$

We have calculated the correlation energy and the expectation value of the correlation potential over a wide range of $\omega_{0}$. This information can serve as constraints in the search for the correlation energy functional, since the correct form of the functional must reproduce both the correlation energy and the correlation-potential expectation value. The dependence of the correlation energy and correlation-potential expectation value on the oscillator frequency $\omega_{0}$ are presented in Fig. 1 for a wide range of $\omega_{0}$. Small values of $\omega_{0}$ correspond to the low-density (and strong correlation) limit while large values of $\omega_{0}$ correspond to the high-density (and weak correlation) limit.

\section{B. Hooke's atom in a laser field: an exact Floquet solution of the two-electron problem}

For the two-electron Hooke's atom model, only the CM equation (60) is affected by the external time-dependent field. Equations (60) and (64) describe a 3D harmonic oscillator subject to a linearly polarized monochromatic field. In this case analytic results also can be obtained. The timeperiodic part $\Phi\left(\mathbf{R}_{C M}, t\right)$ of the quasienergy wave function originating from the ground state of the center-of-mass motion can be expressed as

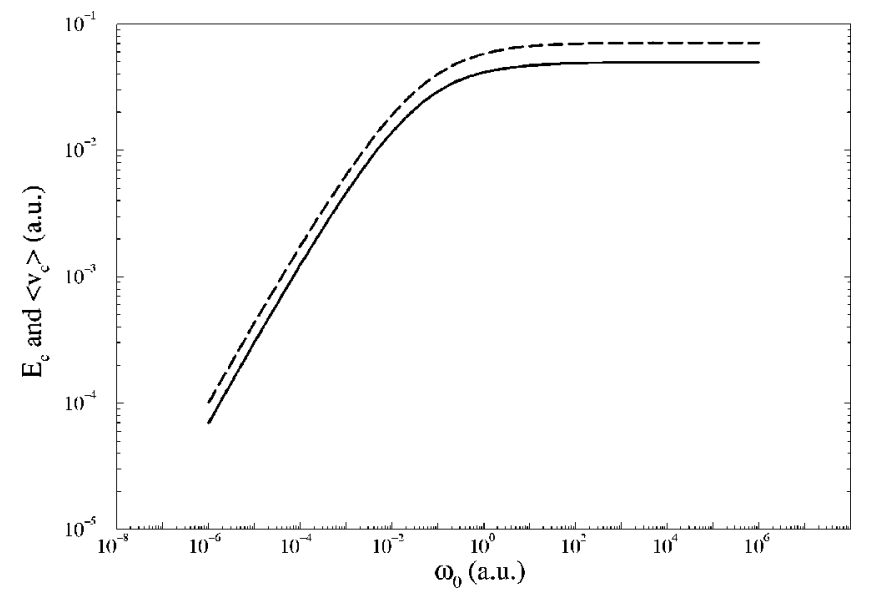

FIG. 1. Absolute values of the correlation energy (solid line) and expectation value of the correlation potential (dashed line) of the field-free Hooke's atom as functions of the oscillator frequency $\omega_{0}$.

$$
\begin{aligned}
\Phi\left(\mathbf{R}_{C M}, t\right)= & \left(\frac{2 \omega_{0}}{\pi}\right)^{3 / 4} \exp \left[-\omega_{0}\left(\mathbf{R}_{C M}-\frac{\mathbf{F}}{\omega_{0}^{2}-\omega^{2}} \cos \omega t\right)^{2}\right. \\
& \left.-i 2 \omega \frac{\left(\mathbf{F} \cdot \mathbf{R}_{C M}\right)}{\omega_{0}^{2}-\omega^{2}} \sin \omega t\right] \\
& \times \exp \left[i \frac{F^{2}\left(\omega_{0}^{2}+\omega^{2}\right)}{4 \omega\left(\omega_{0}^{2}-\omega^{2}\right)^{2}} \sin 2 \omega t\right],
\end{aligned}
$$

and the field-dependent quasienergy $\varepsilon_{C M}$ reads as

$$
\varepsilon_{C M}=\varepsilon_{C M}^{(0)}-\frac{1}{2} F^{2} \frac{1}{\omega_{0}^{2}-\omega^{2}}=\frac{3}{2} \omega_{0}-\frac{1}{2} F^{2} \frac{1}{\omega_{0}^{2}-\omega^{2}} .
$$

Note that the density distribution of the field-driven harmonic oscillator produced by the wave function (102) has the same shape as in the field-free case and oscillates rigidly [harmonic-potential theorem (HPT) [24]]:

$$
\begin{aligned}
\left|\Phi\left(\mathbf{R}_{C M}, t\right)\right|^{2}= & \left(\frac{2 \omega_{0}}{\pi}\right)^{3 / 2} \\
& \times \exp \left[-2 \omega_{0}\left(\mathbf{R}_{C M}-\frac{\mathbf{F}}{\omega_{0}^{2}-\omega^{2}} \cos \omega t\right)^{2}\right] .
\end{aligned}
$$

As a consequence, the one-electron density of the Hooke's atom in the laser field, $\rho(\mathbf{r}, t)$ [see Eq. (67)], has the same property. It can be calculated according to the expression 


$$
\begin{aligned}
\rho(\mathbf{r}, t)= & \frac{8}{r_{F}(t)}\left(\frac{2 \omega_{0}}{\pi}\right)^{1 / 2} \\
& \times \exp \left[-2 \omega_{0} r_{F}^{2}(t)\right] \int_{0}^{\infty} d r^{\prime} r^{\prime} \varphi^{2}\left(r^{\prime}\right) \\
& \times \exp \left(-\frac{1}{2} \omega_{0} r^{\prime 2}\right) \sinh \left[2 \omega_{0} r^{\prime} r_{F}(t)\right],
\end{aligned}
$$

where $r_{F}(t)$ is the absolute value of the time-shifted radius vector,

$$
r_{F}(t)=\left|\mathbf{r}-\frac{\mathbf{F}}{\omega_{0}^{2}-\omega^{2}} \cos \omega t\right|
$$

In other words, the following relation holds:

$$
\rho(\mathbf{r}, t)=\rho^{(0)}\left(r_{F}(t)\right) .
$$

The time-dependent components of the energy can be calculated with the help of the wave function (102) or the density (107). The kinetic energy $T_{C M}(t)$ and one-particle potential energy $U_{C M}(t)$ contributions from the center-of-mass motion are calculated as follows:

$$
\begin{gathered}
T_{C M}(t)=\frac{3}{4} \omega_{0}+\frac{1}{2} F^{2} \frac{\omega^{2}}{\left(\omega_{0}^{2}-\omega^{2}\right)^{2}} \\
-\frac{1}{2} F^{2} \frac{\omega^{2}}{\left(\omega_{0}^{2}-\omega^{2}\right)^{2}} \cos 2 \omega t \\
U_{C M}(t)=\frac{3}{4} \omega_{0}+\frac{1}{2} F^{2} \frac{\omega_{0}^{2}}{\omega_{0}^{2}-\omega^{2}}+\frac{1}{2} F^{2} \frac{\omega_{0}^{2}}{\left(\omega_{0}^{2}-\omega^{2}\right)^{2}} \cos 2 \omega t .
\end{gathered}
$$

Taking into account the time-independent contributions from the relative motion, one obtains the following exact (twoelectron) Hooke's atom kinetic and potential energies in the laser field:

$$
\begin{aligned}
& T(t)=T^{(0)}+\frac{1}{2} F^{2} \frac{\omega^{2}}{\left(\omega_{0}^{2}-\omega^{2}\right)^{2}}-\frac{1}{2} F^{2} \frac{\omega^{2}}{\left(\omega_{0}^{2}-\omega^{2}\right)^{2}} \cos 2 \omega t \\
& U(t)=U^{(0)}+\frac{1}{2} F^{2} \frac{\omega_{0}^{2}}{\left(\omega_{0}^{2}-\omega^{2}\right)^{2}}+\frac{1}{2} F^{2} \frac{\omega_{0}^{2}}{\left(\omega_{0}^{2}-\omega^{2}\right)^{2}} \cos 2 \omega t .
\end{aligned}
$$

In the case of $\omega_{0}=1 / 2$ the field-free values $T^{(0)}$ and $U^{(0)}$ are given by Eqs. (87) and (88), respectively. The expectation value $V_{\text {ext }}(t)$ of the external time-dependent field also has an analytic expression:

$$
V_{\text {ext }}(t)=-F^{2} \frac{1}{\omega_{0}^{2}-\omega^{2}}-F^{2} \frac{1}{\omega_{0}^{2}-\omega^{2}} \cos 2 \omega t
$$

Thus, we present the expectation value $D(t)$ of the timederivative operator $-i \partial / \partial t$ that is important in timedependent problems:

$$
D(t)=-F^{2} \frac{\omega^{2}}{\left(\omega_{0}^{2}-\omega^{2}\right)^{2}}+\frac{1}{2} F^{2} \frac{1}{\omega_{0}^{2}-\omega^{2}} \cos 2 \omega t .
$$

The electron-electron interaction energy $V_{e e}$ is time independent since it depends on the relative motion only, which is not affected by the external field:

$$
V_{e e}=V_{e e}^{(0)} \text {. }
$$

In the case $\omega_{0}=1 / 2$ it is given by Eq. (89).

Note that dependence on time of the expectation values of kinetic energy, potential energy, external field, and timederivative operator is confined to the function $\cos 2 \omega t$. This is inherent to the harmonic oscillator behavior in the external monochromatic field.

One can also calculate various combinations of the quantities $T(t), U(t), V_{e x t}(t)$, and $V_{e e}$. For example, the timedependent expectation value of the total Hamiltonian $H$ [the time-dependent energy $E(t)$ of the Hooke's atom] is expressed as

$$
\begin{aligned}
E(t)= & T(t)+U(t)+V_{e e}+V_{e x t}(t) \\
= & \varepsilon_{R M}+\varepsilon_{C M}^{(0)}-\frac{1}{2} F^{2} \frac{\omega_{0}^{2}-3 \omega^{2}}{\left(\omega_{0}^{2}-\omega^{2}\right)^{2}} \\
& -\frac{1}{2} F^{2} \frac{1}{\omega_{0}^{2}-\omega^{2}} \cos 2 \omega t .
\end{aligned}
$$

Other important combinations are $T(t)+U(t)$ and $T(t)$ $-U(t)$ :

$$
\begin{aligned}
T(t)+U(t)= & T^{(0)}+U^{(0)}+\frac{1}{2} F^{2} \frac{\omega_{0}^{2}+\omega^{2}}{\left(\omega_{0}^{2}-\omega^{2}\right)^{2}} \\
& +\frac{1}{2} F^{2} \frac{1}{\omega_{0}^{2}-\omega^{2}} \cos 2 \omega t, \\
T(t)-U(t)= & T_{r}-U_{r}-\frac{1}{2} F^{2} \frac{1}{\omega_{0}^{2}-\omega^{2}} \\
& -\frac{1}{2} F^{2} \frac{\omega_{0}^{2}+\omega^{2}}{\left(\omega_{0}^{2}-\omega^{2}\right)^{2}} \cos 2 \omega t .
\end{aligned}
$$

Note that the combination $E(t)+D(t)=T(t)+U(t)+V_{e e}$ $+V_{\text {ext }}(t)+D(t)$ is time independent and equal to the total quasienergy $\varepsilon$ :

$$
\begin{aligned}
T(t) & +U(t)+V_{e e}+V_{e x t}(t)+D(t) \\
& =\varepsilon=\varepsilon_{R M}+\varepsilon_{C M}^{(0)}-\frac{1}{2} F^{2} \frac{1}{\omega_{0}^{2}-\omega^{2}} .
\end{aligned}
$$




\section{Hooke's atom in a laser field: construction of the exact}

Kohn-Sham orbital and exchange-correlation potential

In this section, using the exact Floquet solution of the two-electron problem described in Sec. III B, we calculate the exact Kohn-Sham orbital and invert the time-dependent Kohn-Sham equation to obtain the exact exchangecorrelation potential. The time-periodic Kohn-Sham orbital $\phi_{K S}(\mathbf{r}, t)$ must satisfy the quasienergy Kohn-Sham equation:

$$
\begin{aligned}
& {\left[-\frac{1}{2} \nabla^{2}+\frac{1}{2} \omega_{0}^{2} r^{2}-(\mathbf{F} \cdot \mathbf{r}) \cos \omega t+v_{S}(\mathbf{r}, t)-i \frac{\partial}{\partial t}\right]} \\
& \quad \times \phi_{K S}(\mathbf{r}, t)=\epsilon_{K S} \phi_{K S}(\mathbf{r}, t) .
\end{aligned}
$$

Here $\epsilon_{K S}$ is the orbital quasienergy and $v_{s}(\mathbf{r}, t)$ is the timedependent single-particle potential, which includes the Hartree and exchange-correlation potentials:

$$
\begin{gathered}
v_{s}(\mathbf{r}, t)=v_{H}(\mathbf{r}, t)+v_{x c}(\mathbf{r}, t), \\
v_{H}(\mathbf{r}, t)=\int d^{3} r^{\prime} \frac{\rho\left(\mathbf{r}^{\prime}, t\right)}{\left|\mathbf{r}-\mathbf{r}^{\prime}\right|}
\end{gathered}
$$

In the time-dependent problem, the phase of the complex Kohn-Sham orbital $\phi_{K S}(\mathbf{r}, t)$ is important. Let the function $\phi_{K S}(\mathbf{r}, t)$ be represented through its modulus and phase:

$$
\phi_{K S}(\mathbf{r}, t)=M(\mathbf{r}, t) \exp [i P(\mathbf{r}, t)] .
$$

Upon substitution of Eq. (122) in Eq. (119), one obtains the following two coupled equations to solve for the modulus and phase:

$$
\begin{gathered}
-\frac{1}{2}\left[\nabla^{2} M-M(\nabla P)^{2}\right]+M \frac{\partial P}{\partial t} \\
+\left[\frac{1}{2} \omega_{0}^{2} r^{2}-(\mathbf{F} \cdot \mathbf{r}) \cos \omega t+v_{s}(\mathbf{r}, t)\right] M=\epsilon_{K S} M, \\
\frac{\partial M^{2}}{\partial t}+\left[\nabla \cdot\left(M^{2} \nabla P\right)\right]=0 .
\end{gathered}
$$

Our goal is to calculate both the modulus and phase of the Kohn-Sham orbital and then obtain the time-dependent single-particle potential $v_{s}(\mathbf{r}, t)$. As one will see, the results can be obtained in the analytic form if the RM problem has an analytic solution. At least, all the quantities can be expressed through the (time-independent, spherically symmetric) $\mathrm{RM}$ wave function, and the time dependence of the expectation values can be obtained analytically.

First, the modulus $M(\mathbf{r}, t)$ is easily obtained from the oneelectron density (105),

$$
M(\mathbf{r}, t)=\left[\frac{1}{2} \rho(\mathbf{r}, t)\right]^{1 / 2},
$$

and, taking into account Eqs. (107) and (71), can be expressed through the unperturbed Kohn-Sham orbital $\phi_{K S}^{(0)}$ calculated at the shifted variable $r_{F}(t)$,

$$
M(\mathbf{r}, t)=\phi_{K S}^{(0)}\left(r_{F}(t)\right)
$$

where $r_{F}(t)$ is given in Eq. (106). Then the phase $P(\mathbf{r}, t)$ can be calculated from Eq. (124). This is a general continuity equation but because of the specific dependence of the function $M(\mathbf{r}, t)$ on time (through the variable $r_{F}$ ), it can be recast in the following form:

$$
\left\{\boldsymbol{\nabla} \cdot\left[M^{2}\left(\boldsymbol{\nabla} P+\mathbf{F} \frac{\omega}{\omega_{0}^{2}-\omega^{2}} \sin \omega t\right)\right]\right\}=0 .
$$

The particular solution of Eq. (127) that serves the purpose is as follows:

$$
P(\mathbf{r}, t)=-(\mathbf{F} \cdot \mathbf{r}) \frac{\omega}{\omega_{0}^{2}-\omega^{2}} \sin \omega t+f(t)
$$

The unknown function $f(t)$, which depends on the time only, cannot be determined from the continuity equation. The particular choice of this term can be made when inserting the phase $P(\mathbf{r}, t)$ in Eq. (123). The requirement that the potential $v_{s}(\mathbf{r}, t)$ vanishes as $r \rightarrow \infty$ determines both the term $f(t)$ and the quasienergy $\epsilon_{K S}$. The final expressions for the timedependent Kohn-Sham orbital $\phi_{K S}(\mathbf{r}, t)$ and quasienergy $\epsilon_{K S}$ are as follows:

$$
\begin{gathered}
\phi_{K S}(\mathbf{r}, t)=\phi_{K S}^{(0)}\left(r_{F}(t)\right) \\
\times \exp \left[-i \omega \frac{(\mathbf{F} \cdot \mathbf{r})}{\omega_{0}^{2}-\omega^{2}} \sin \omega t\right. \\
\left.+i \frac{F^{2}\left(\omega_{0}^{2}+\omega^{2}\right)}{8 \omega\left(\omega_{0}^{2}-\omega^{2}\right)^{2}} \sin 2 \omega t\right], \\
\epsilon_{K S}=\epsilon_{K S}^{(0)}-\frac{1}{4} F^{2} \frac{1}{\omega_{0}^{2}-\omega^{2}}=\varepsilon_{R M}-\frac{1}{4} F^{2} \frac{1}{\omega_{0}^{2}-\omega^{2}} .
\end{gathered}
$$

When employing the calculated modulus and phase of the Kohn-Sham orbital in Eq. (123), another important conclusion can be made regarding the single-particle potential $v_{s}(\mathbf{r}, t)$, namely, it can be expressed by means of the unperturbed single-particle potential $v_{s}^{(0)}$ calculated at the shifted independent variable $r_{F}(t)$ :

$$
v_{s}(\mathbf{r}, t)=v_{s}^{(0)}\left(r_{F}(t)\right) \text {. }
$$

Since the Hartree potential $v_{H}(\mathbf{r}, t)$ defined by Eq. (121) obviously satisfies the similar equation

$$
v_{H}(\mathbf{r}, t)=v_{H}^{(0)}\left(r_{F}(t)\right)
$$

due to the corresponding property of the density [see Eq. (107)], it follows that the exchange-correlation potential $v_{x c}(\mathbf{r}, t)$ alone also must satisfy a similar equation:

$$
v_{x c}(\mathbf{r}, t)=v_{x c}^{(0)}\left(r_{F}(t)\right) .
$$




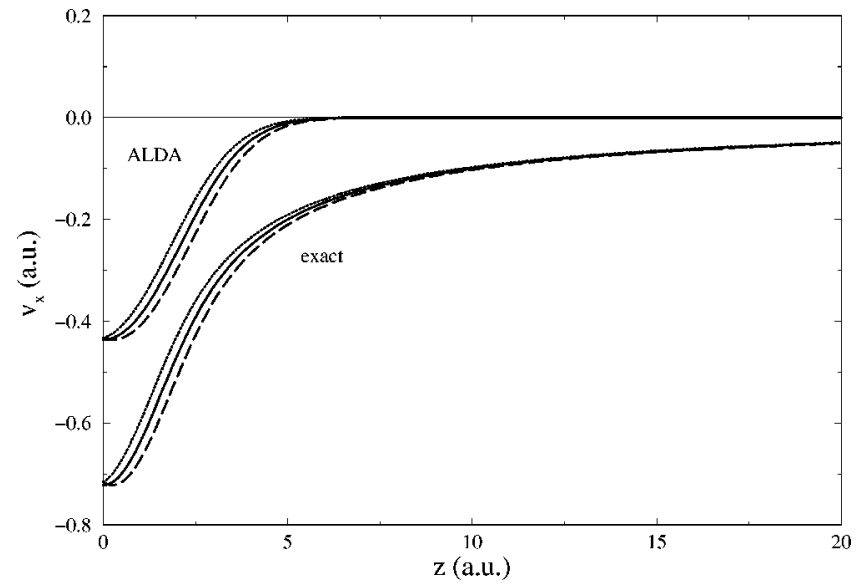

FIG. 2. Exact and ALDA exchange potentials for the Hooke's atom at $\omega_{0}=1 / 2$ a.u. as measured along the positive part of the $z$ axis (external field is polarized in the $z$ direction) at different time moments. The solid lines correspond to the zero instantaneous field, $\cos \omega t=0$, while the dotted and dashed lines correspond to the peak values of the field, $\cos \omega t=-1$ and $\cos \omega t=1$, respectively.

This is again a manifestation of the HPT [24]. The exact exchange-correlation potential satisfies Eq. (133) as shown by its construction. Equation (133) serves as a useful constraint and test of the accuracy and applicability of the approximate time-dependent exchange-correlation potential form used. For example, if the approximate exchangecorrelation potential used is a local functional of the density [as in ALDA, $\left.v_{x c}(\mathbf{r}, t)=v_{x c}(\rho(\mathbf{r}, t))\right]$, it will have the same functional form as for the unperturbed (time-independent) system but calculated with the shifted density $\rho^{(0)}\left(r_{F}(t)\right)$, i.e., the density $\rho(\mathbf{r}, t)$ of the system in the field. However, in general the exact functional can be nonlocal in both space and time; the possible forms of the exchange-correlation potential with memory that satisfy Eq. (133) and other exact symmetries were studied in Ref. [17]. An investigation of the adiabatic and dynamic contributions to the exchangecorrelation potential in a model involving the 2D Hooke's atom (but not for HPT motion) [25] showed that dynamic contributions were negligibly small.

In Figs. 2 and 3 we compare exact and ALDA [26] exchange and correlation potentials, respectively, at different time moments. The oscillator frequency used in the calculations is $\omega_{0}=1 / 2$ a.u. and the laser field parameters are as follows: $\omega=0.22$ a.u. and $F=0.05$ a.u. This is a rather strong external field and the potential curves at different time moments (dotted line for $\cos \omega t=-1$ and dashed line for $\cos \omega t=1)$ are displaced significantly from that of the zero instantaneous field $(\cos \omega t=0)$. The ALDA potentials are obtained directly from extension of the static LDA form [26] to the time domain using the adiabatic approximation. For the exchange potential at large $r$, the ALDA curve decays to zero exponentially, while the exact potential follows the correct $-1 / r$ Coulombic behavior. There is also substantial difference in the short-range part between the two potentials. Similarly, there is significant difference in the two correlation potentials. Such discrepancies are expected and can be attributed to an intrinsic defect of the LDA exchange-

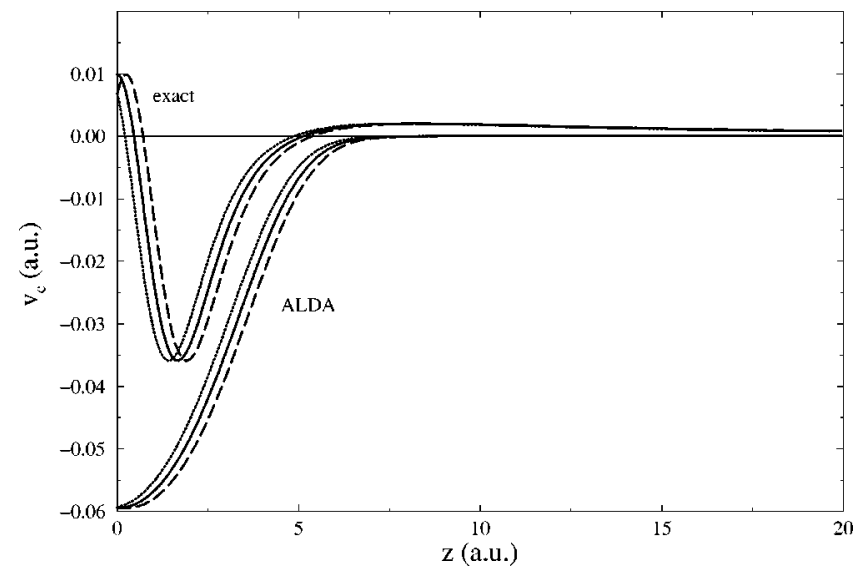

FIG. 3. Exact and ALDA correlation potentials for the Hooke's atom at $\omega_{0}=1 / 2$ a.u. as measured along the positive part of the $z$ axis (external field is polarized in the $z$ direction) at different time moments. The curve notations are the same as those in Fig. 2.

correlation potential in its static form, namely, the existence of the self-interaction term in the LDA energy functional. Similar problems still exist even when one uses the more refined generalized gradient approximation [3] in the adiabatic approximation.

In the study of time-dependent dynamics involving the excited and continuum states, it is essential that the longrange exchange-correlation potential be treated more accurately. The recent development of time-dependent optimized effective potential (OEP) methods [27,28] based on extension of the KLI (Krieger-Li-Iafrate) theory [29] to the time domain has significantly advanced this field. If an explicit self-interaction-correction (SIC) form is further introduced, the time-dependent OEP/KLI-SIC procedure leads to a single-particle exchange-correlation potential that is both local and orbital independent and has the proper long-range Coulombic behavior [28]. For the special case of twoelectron systems, the OEP/KLI procedure in fact reduces to the exact Hartree-Fock potential (for the exchange part). Applying this time-dependent OEP/KLI-SIC procedure to the present problem, we recover the exact time-dependent exchange potential as shown in Fig. 2.

Now we proceed to the calculation of the various contributions to the quasienergy functional of the Kohn-Sham system. The noninteracting kinetic energy $T_{s}(t)$ defined as

$$
T_{s}(t)=2\left\langle\phi_{K S}(\mathbf{r}, t)\left|-\frac{1}{2} \nabla^{2}\right| \phi_{K S}(\mathbf{r}, t)\right\rangle
$$

is determined with the help of Eq. (129), giving

$$
T_{s}(t)=T_{s}^{(0)}+\frac{1}{2} F^{2} \frac{\omega^{2}}{\left(\omega_{0}^{2}-\omega^{2}\right)^{2}}-\frac{1}{2} F^{2} \frac{\omega^{2}}{\left(\omega_{0}^{2}-\omega^{2}\right)^{2}} \cos 2 \omega t .
$$

Note that the dependence on the external field and time in $T_{s}(t)$ are the same as in the exact two-electron kinetic energy $T(t)$ [Eq. (110)]. Thus the correlation kinetic energy $T_{c}$ is time independent and coincides with its unperturbed value:

$$
T_{c}(t)=T(t)-T_{s}(t)=T^{(0)}-T_{s}^{(0)}=T_{c}^{(0)} .
$$


The expectation value $D_{s}(t)$ of the noninteracting timederivative operator is calculated according to

$$
D_{s}(t)=2\left\langle\phi_{K S}(\mathbf{r}, t)\left|i \frac{\partial}{\partial t}\right| \phi_{K S}(\mathbf{r}, t)\right\rangle \text {. }
$$

It is found that $D_{s}(t)$ is the same as the two-electron expectation value $D(t)$ :

$$
D_{s}(t)=F^{2} \frac{\omega^{2}}{\left(\omega_{0}^{2}-\omega^{2}\right)^{2}}-\frac{1}{2} F^{2} \frac{1}{\omega_{0}^{2}-\omega^{2}} \cos 2 \omega t=D(t) \text {. }
$$

The Hartree energy $J(t)$ is defined according to

$$
\begin{aligned}
J(t) & =\frac{1}{2} \int d^{3} r \int d^{3} r^{\prime} \frac{\rho(\mathbf{r}, t) \rho\left(\mathbf{r}^{\prime}, t\right)}{\left|\mathbf{r}-\mathbf{r}^{\prime}\right|} \\
& =\frac{1}{2} \int d^{3} r \rho(\mathbf{r}, t) v_{H}(\mathbf{r}, t) .
\end{aligned}
$$

Since the density $\rho(\mathbf{r}, t)$ and Hartree potential $v_{H}(\mathbf{r}, t)$ possess the properties (107) and (132), respectively, the integral in the right-hand side of Eq. (139) becomes time independent, and the Hartree energy coincides with its unperturbed value:

$$
J(t)=J^{(0)} .
$$

Similarly, the exchange-correlation energy $E_{x c}(t)$ defined according to Eq. (19) also reduces to its unperturbed value:

$$
E_{x c}(t)=E_{x c}^{(0)} .
$$

The results (135) and (138), yielding the time dependence of the noninteracting kinetic energy and time derivative, and (136), (140), and (141), demonstrating actual time independence of the correlation kinetic energy, Hartree energy, and exchange-correlation energy, can be attributed to the special model under consideration (HPT motion). In particular, that the Hartree and exchange-correlation energies do not actually depend on time is not true in the more general case.

As in the field-free case (see Sec. III A), one can split the exchange-correlation energy into exchange-only (HartreeFock) and correlation parts. The further analysis here is essentially the same as in Sec. III A since all the expectation values related to the exchange and correlation potentials reduce to the unperturbed ones.

To apply the general time-dependent relations (37) and (38), note that for HPT motion the following equations hold:

$$
\begin{aligned}
& \frac{\partial v_{H}(\mathbf{r}, t)}{\partial t}=\left[\mathbf{F} \cdot \nabla v_{H}^{(0)}\left(r_{F}\right)\right] \frac{\omega}{\omega_{0}^{2}-\omega^{2}} \sin \omega t, \\
& \frac{\partial v_{x c}(\mathbf{r}, t)}{\partial t}=\left[\mathbf{F} \cdot \nabla v_{x c}^{(0)}\left(r_{F}\right)\right] \frac{\omega}{\omega_{0}^{2}-\omega^{2}} \sin \omega t .
\end{aligned}
$$

Then in the right-hand side of Eq. (37) the integrals containing the time derivatives of the Hartree and exchange- correlation potentials vanish due to exact symmetry (the total interparticle repulsion and exchange-correlation forces are equal to zero by Newton's third law). The remaining integral containing the time derivative of the external field is exactly the same as in Eq. (27), so the time dependence of $D(t)$ and $D_{s}(t)$ is the same [actually, for HPT motion, the entire expectation values $D_{s}(t)$ and $D(t)$ coincide; see Eq. (138)]. In the right-hand side of Eq. (38) the integrals containing the exchange-correlation and Hartree potentials are expressed through the integrals appearing in Eq. (37) and the time derivatives of the expectation values of exchange-correlation and Hartree potentials, respectively,

$$
\begin{aligned}
\int d^{3} r \frac{\partial \rho(\mathbf{r}, t)}{\partial t} v_{H}(\mathbf{r}, t)= & \frac{d}{d t} \int d^{3} r \rho(\mathbf{r}, t) v_{H}(\mathbf{r}, t) \\
& -\int d^{3} r \rho(\mathbf{r}, t) \frac{\partial v_{H}(\mathbf{r}, t)}{\partial t} \\
\int d^{3} r \frac{\partial \rho(\mathbf{r}, t)}{\partial t} v_{x c}(\mathbf{r}, t)= & \frac{d}{d t} \int d^{3} r \rho(\mathbf{r}, t) v_{x c}(\mathbf{r}, t) \\
& -\int d^{3} r \rho(\mathbf{r}, t) \frac{\partial v_{x c}(\mathbf{r}, t)}{\partial t}
\end{aligned}
$$

Since the expectation values of the Hartree and exchangecorrelation potentials are time independent for HPT motion, the time derivatives of $T_{s}$ and $T$ are the same, in accordance with Eqs. (110) and (135). To confirm the general relation (39) regarding the exchange-correlation energy, one needs only to make sure that the quasienergy combination appearing on the right-hand side of Eq. (39) is field independent, since all the expectation values reduce to the unperturbed ones. With the help of Eqs. (118) and (130), one can see that this is the case, so the general relation (39) holds.

In conclusion, we have presented in this paper several exact relations that hold in the Floquet formulation of TDDFT. The most important results are those involving the time-dependent exchange-correlation energy and potential, Eqs. (39), (40), and (50). They can be used as constraints for testing the validity and accuracy of approximate forms of the time-dependent exchange-correlation functionals. The general relations are verified in an exactly soluble model, the two-electron Hooke's atom in a linearly polarized laser field. This model exhibits a special kind of motion, described by the harmonic potential theorem [24]. Further work including the consideration of models beyond the HPT would allow a more complete investigation of the time-dependent properties of the exchange-correlation energy functionals and potentials. Work in this direction is in progress.

\section{ACKNOWLEDGMENT}

We are grateful to the US Department of Energy, Office of Science, Office of Basic Energy Sciences, Division of Chemical Sciences for partial support of this work. 
[1] P. Hohenberg and W. Kohn, Phys. Rev. 136, B864 (1964).

[2] W. Kohn and L. J. Sham, Phys. Rev. 140, A1113 (1965).

[3] See, e.g., R. G. Parr and W. Yang, Density-Functional Theory of Atoms and Molecules (Oxford University Press, Oxford, 1989); R. M. Dreizler and E. K. U. Gross, Density Functional Theory, An Approach to the Quantum Many-Body Problem (Springer, Berlin, 1990); Density Functional Theory Vol. 337 of Advanced Study NATO Institute, Series B: Physics, edited by E. K. U. Gross and R. M. Dreizler (Plenum Press, New York, 1995); N. H. March, Electron Density Theory of Atoms and Molecules (Academic Press, San Diego, 1992); Density Functional Methods in Chemistry, edited by J. K. Labanowski and J. W. Andzelm (Springer, Berlin, 1991).

[4] A. Zangwill and P. Soven, Phys. Rev. A 21, 1561 (1980).

[5] B. M. Deb and S. K. Ghosh, J. Chem. Phys. 77, 342 (1982).

[6] L. J. Bartolotti, Phys. Rev. A 24, 1661 (1981); 26, 2243 (1982).

[7] E. Runge and E. K. U. Gross, Phys. Rev. Lett. 52, 997 (1984).

[8] A. K. Rajagopal, Phys. Rev. A 50, 3759 (1994).

[9] S. K. Ghosh and A. K. Dhara, Phys. Rev. A 38, 1149 (1988).

[10] S. M. Colwell, N. C. Handy, and A. M. Lee, Phys. Rev. A 53, 1316 (1996).

[11] D. A. Telnov and S. I. Chu, Chem. Phys. Lett. 264, 466 (1997).

[12] D. A. Telnov and S. I. Chu, Phys. Rev. A 58, 4749 (1998).

[13] D. A. Telnov and S. I. Chu, Int. J. Quantum Chem. 69, 305 (1998).
[14] E. K. U. Gross and W. Kohn, Phys. Rev. Lett. 55, 2850 (1985).

[15] G. Vignale and W. Kohn, Phys. Rev. Lett. 77, 2037 (1996).

[16] G. Vignale, C. Ullrich, and S. Conti, Phys. Rev. Lett. 79, 4878 (1997).

[17] J. F. Dobson, M. J. Bünner, and E. K. U. Gross, Phys. Rev. Lett. 79, 1905 (1997).

[18] P. Hessler, J. Park, and K. Burke, Phys. Rev. Lett. 82, 378 (1999).

[19] P. M. Laufer and J. B. Krieger, Phys. Rev. A 33, 1480 (1986).

[20] C. Filippi, C. J. Umrigar, and M. Taut, J. Chem. Phys. 100, 1290 (1994).

[21] S. Kais, D. R. Herschbach, and R. D. Levine, J. Chem. Phys. 91, 7791 (1989).

[22] S. Kais, D. R. Herschbach, N. C. Handy, C. W. Murray, and G. J. Laming, J. Chem. Phys. 99, 417 (1993).

[23] M. Taut, Phys. Rev. A 48, 3561 (1993).

[24] J. F. Dobson, Phys. Rev. Lett. 73, 2244 (1994).

[25] I. D’Amico and G. Vignale, Phys. Rev. B 59, 7876 (1999).

[26] J. P. Perdew and Y. Wang, Phys. Rev. B 45, 13244 (1992).

[27] C. A. Ullrich, U. J. Gossmann, and E. K. U. Gross, Phys. Rev. Lett. 74, 872 (1995).

[28] X. M. Tong and S. I. Chu, Phys. Rev. A 57, 452 (1998); X. Chu and S. I. Chu, in Multiphoton Processes: ICOMP VIII, edited by L. F. DiMauro, R. R. Freeman, and K. C. Kulander (American Institute of Physics, Melville, NY, 2000), pp. 415426.

[29] J. Krieger, Y. Li, and G. Iafrate, Phys. Rev. A 46, 5453 (1992). 